\title{
Quasilinear evolution of cyclotron maser instability
}

\author{
Peter H. Yoon \\ Institute for Physical Science and Technology, University of Maryland, College Park Maryland 20742 \\ L. F. Ziebell \\ Instituto de Física, Universidade Federal do Rio Grande do Sul, Caixa Postal 15051, 91501-970 Porto Alegre, RS, Brazil
}

(Received 21 September 1994)

\begin{abstract}
A quasilinear analysis of the relativistic electron cyclotron maser instability is presented. A background plasma is assumed to support the wave motion, while the instability is driven by a tenuous population of energetic electrons possessing a loss-cone feature. The analysis makes use of an efficient moment method. In this approach, evolution equations for the moments of particle distribution function are derived from the particle kinetic equation. Then, a self-similar model of the loss-cone electron distribution function is imposed. Simultaneously, the wave kinetic equation is solved. The resulting fully self-consistent set of equations that governs the evolution of the particles and unstable waves is solved numerically under physical parameters that represent typical solar microwave burst sources.
\end{abstract}

PACS number(s): 52.35.-g

\section{INTRODUCTION}

The cyclotron maser instability is driven by a losscone feature in the electron distribution function (or more precisely, by an inverted population in momentum space perpendicular to the magnetic field, hence the term maser). The instability mechanism excites a variety of wave modes in the vicinity of the electron cyclotron frequency and/or its harmonics, including the fast electromagnetic mode. The excitation of fast waves leads to direct amplification of radiation. Therefore the maser instability is an important radiation mechanism with a wide range of applicability in many astrophysical radio sources, planetary and solar radio emissions, and laboratory microwave generation devices. The instability cannot be described under the nonrelativistic formalism despite the fact that, in many applications, typical particle energy is nonrelativistic. Therefore it is called the relativistic electron cyclotron maser in the literature.

Over the past decade or so, an extensive body of literature on the subject of the cyclotron maser has been accumulated [1-28]. Many articles in the literature emphasize the linear aspect of the instability, although a few discussions concerning nonlinear aspects can be found. Most of the nonlinear theories make use of the numerical simulation method. The results from these simulations led to the conclusion that the dominant saturation mechanism for the maser instability is quasilinear saturation of unstable waves with concomitant filling up of the loss cone. Therefore it is appropriate to study the nonlinear stage of the instability by employing quasilinear kinetic theory.

In the past, some attempts have been made to employ quasilinear theory to the maser instability $[17,19,24,26]$. In particular, Refs. [24,26] discuss calculations of instantaneous anomalous transport rates that result from maser process, while Ref. [17] discusses the solution of the quasilinear kinetic equation by assuming a quasiperpendicular or exactly perpendicular propagation angle. A recent work by Aschwanden [24], however, makes an important step toward a comprehensive treatment of the cyclotron maser instability within the context of quasilinear theory. In Ref. [24] a set of fully self-consistent particle and wave kinetic equations is numerically solved by assuming that the fundamental $\mathrm{X}$ mode prevails over other modes. The analysis was carried out with specific application to the solar microwave burst phenomenon.

In this paper we describe an alternative method for a quasilinear analysis similar to that in Ref. [24]. The method we introduce involves the use of an efficient moment method under an assumed model time-dependent particle distribution function which has a self-similar form. The construction of the model distribution function is guided by the fact that in the nonlinear stage of the instability the dominant feature in the particle evolution will be the filling up of the loss cone. This procedure greatly reduces the computational time, although it may not be strictly rigorous in a mathematical sense. Nevertheless, with this method, we demonstrate that one can obtain reliable physical results very effectively. To test the reliability of the present approach, we make a direct comparison with the more formal results obtained in Ref. [24]. Indeed, it is found that results obtained with this approach agree well with those of Ref. [24]. Furthermore, we also consider a parameter regime not considered in Ref. [24], and conclude that the competition between different unstable modes must be incorporated for a general situation, and that large initial growth rate does not necessarily mean that that particular mode will prevail over other modes with smaller growth rates in the later stage of evolution.

This paper is organized in the following manner. In Sec. II the linear analysis of the maser instability is per- 
formed. Then Sec. III discusses the nonlinear stage of the instability with the use of the aforementioned moment method. Finally, Sec. IV presents the conclusions and discussion.

\section{LINEAR STAGE OF THE MASER INSTABILITY}

The present analysis is concerned with high frequency waves. Therefore the ions are treated as a neutralizing background. The electrons are assumed to be comprised of a relatively less energetic component and an energetic species possessing a (one-sided) loss-cone feature in momentum space. The physical reason for the one sidedness of the loss cone is the following. The cyclotron maser emission is the source of Earth's auroral kilometric radiation (AKR) [1] and the solar microwave bursts [24], among other examples. In these cases, the loss-cone feature in the energetic electron population is a result of diverging ambient magnetic field with monotonically decreasing strength, which reflects those electrons traveling from a weak-field region to regions with increasing field strength, hence the one-sided loss-cone.

In the present analysis, it is assumed that the density of the background component is sufficiently higher than that of the energetic component so that the dispersion relation can be determined from the background component while the energetic component gives rise to the wave growth. For the case of the Earth's AKR, this assumption is not appropriate. In fact, in the AKR source, the loss-cone electrons may dominate over the cold component. (In a concurrent article [32], the AKR case is addressed.) However, for solar microwave bursts, this assumption is valid [24]. Therefore, in the present analysis, we consider physical parameters appropriate for the solar case.

As is customary, the ambient magnetic field is assumed to lie in the $z$ direction $\left(\mathbf{B}_{0}=B_{0} \mathbf{e}_{z}\right)$, and the wave vector $\mathbf{k}$ lies in the $x z$ plane, $\mathbf{k}=\mathbf{e}_{x} k \sin \theta+\mathbf{e}_{z} k \cos \theta$. In determining the real frequency, background thermal effects are ignored.

The following cold-plasma (or, magnetoionic) dispersion relation is well known [29-31]:

$$
\begin{aligned}
N^{2} & =1-\frac{T \omega_{p}^{2}}{\omega(T \omega-\Omega \cos \theta)}, \\
T & =\frac{\omega_{p}^{2}-\omega^{2}}{\left|\omega_{p}^{2}-\omega^{2}\right|}\left[s-\sigma\left(1+s^{2}\right)^{\frac{1}{2}}\right], \\
s & =\frac{\omega \Omega \sin ^{2} \theta}{2\left|\omega^{2}-\omega_{p}^{2}\right| \cos \theta},
\end{aligned}
$$

where $\sigma=+1$ for the $\mathrm{X}$ mode, and $\sigma=-1$ for the $\mathrm{O}$ mode. (The definitions for the $\mathrm{X}$ and $\mathrm{O}$ modes are well known, and thus are not repeated here.) In Eq. (1), $N=c k / \omega$ is the index of refraction; $\Omega=e B_{0} / m c$ is the electron cyclotron frequency, $e$ being the electron charge, $m$ being the electron mass, and $c$ being the speed of light in vacuo; and $\omega_{p}^{2}=4 \pi n_{0} e^{2} / m$ is the square of the electron plasma frequency, $n_{0}$ being the number density associated with the background electrons.
Often, the $\mathrm{X}$ mode is further classified into $\mathrm{X}$ and $\mathrm{Z}$ modes, where these are defined by their respective frequency ranges: $\omega>\omega_{\mathrm{X}}$ for the $\mathrm{X}$ mode, while $\omega_{\mathrm{O}}<$ $\omega<\omega_{\mathrm{Z}}$ for the $\mathrm{Z}$ mode. Similarly, the $\mathrm{O}$ mode is also further distinguished by respective frequency ranges into $\mathrm{O}$ and $\mathrm{W}$ modes. These are defined by $\omega>\omega_{p}$ and $0<\omega<\omega_{\mathrm{W}}$, respectively. Here, $\omega_{\mathrm{X}}$ and $\omega_{\mathrm{O}}$ are cutoff frequencies defined, respectively, by $\omega_{\mathrm{X}}=$ $\left[\left(\Omega^{2}+4 \omega_{p}^{2}\right)^{1 / 2}+\Omega\right] / 2$ and $\omega_{\mathrm{O}}=\left[\left(\Omega^{2}+4 \omega_{p}^{2}\right)^{1 / 2}-\Omega\right] / 2$. Further, $\omega_{\mathrm{Z}}$ and $\omega_{\mathrm{W}}$ are resonance frequencies defined by $\omega_{\mathrm{Z}}=(1 / \sqrt{2})\left[\omega_{H}^{2}+\left(\omega_{H}^{4}-4 \omega_{p}^{2} \Omega^{2} \cos ^{2} \theta\right)^{1 / 2}\right]^{1 / 2}$ and $\omega_{\mathrm{W}}=(1 / \sqrt{2})\left[\omega_{H}^{2}-\left(\omega_{H}^{4}-4 \omega_{p}^{2} \Omega^{2} \cos ^{2} \theta\right)^{1 / 2}\right]^{1 / 2}$, respectively. Here, $\omega_{H}=\left(\omega_{p}^{2}+\Omega^{2}\right)^{1 / 2}$ is the familiar upperhybrid frequency.

In evaluating the linear growth rate for the maser instability, we employ the weakly (or, semi) relativistic approximation. That is, we replace the relativistic Lorentz factor $\gamma=\left(1+p^{2} / m^{2} c^{2}\right)^{1 / 2}$ by 1 everywhere, except when it appears in the wave-particle resonance condition, where it is replaced by $1+p^{2} / 2 m^{2} c^{2}$. In what follows, we introduce a normalized momentum variable, $\mathbf{u}=\mathbf{p} / m c$. Then, the linear growth rate expression can be shown to reduce to $[31]$

$$
\begin{aligned}
& \frac{\gamma_{\mathbf{k}}}{\Omega}=\frac{\pi}{2 R} \frac{\omega_{p}^{2}}{\Omega^{2}} \sum_{n=1}^{\infty} \int d^{3} u \delta\left(1+\frac{u^{2}}{2}-\frac{n \Omega}{\omega}-N u \mu \cos \theta\right) \\
& \times\left([K \sin \theta+T(\cos \theta-N u \mu)] J_{n}(b)+\frac{\Omega}{\omega} b J_{n}^{\prime}(b)\right)^{2} \\
& \times \frac{1}{u}\left(\frac{\partial f}{\partial u}+\frac{N u \cos \theta-\mu}{u} \frac{\partial f}{\partial u}\right), \\
& R(\omega, \theta)=\left(1+T^{2}\right) N^{2} \sin ^{2} \theta\left[\frac{\omega}{\Omega}+\frac{1}{2} \frac{T \omega_{p}^{2} \cos \theta}{(T \omega-\Omega \cos \theta)^{2}}\right. \\
& \left.\times\left(1+\frac{1-T^{2}}{1+T^{2}} \frac{\omega^{2}+\omega_{p}^{2}}{\omega^{2}-\omega_{p}^{2}}\right)\right] \\
& K=\frac{\omega_{p}^{2}}{\omega^{2}-\omega_{p}^{2}} \frac{T \Omega \sin \theta}{T \omega-\Omega \cos \theta}, \\
& b=\frac{\omega}{\Omega} N u\left(1-\mu^{2}\right)^{\frac{1}{2}} \sin \theta .
\end{aligned}
$$

In Eq. (2), $\mu$ is the cosine of the momentum pitch angle, and $f(u, \mu)$ is the total (background plus energetic) electron momentum distribution function.

The background component is modeled by an isotropic Maxwellian distribution, while the energetic component possessing a loss-cone feature is modeled as shown below:

$$
\begin{aligned}
f(u, \mu)= & \frac{1}{\pi^{3 / 2} \alpha_{0}^{3}} \exp \left(-\frac{u^{2}}{\alpha_{0}^{2}}\right) \\
& +\frac{n_{e}}{n_{0}} \frac{1}{\pi^{3 / 2} \alpha^{3}} \exp \left(-\frac{u^{2}}{\alpha^{3}}\right) G(\mu) \\
G(\mu)= & \frac{1}{1+\Delta-\delta \tanh 1 / \delta}\left(\tanh ^{2} \frac{\mu-1}{2 \delta}+\Delta\right)
\end{aligned}
$$


In the above model, $n_{e}$ is the density associated with the energetic component, which is assumed to be much lower than that of the isotropic component $\left(n_{e} \ll n_{0}\right)$. The parameter $\Delta$ ranges from 0 to $\infty$. When $\Delta=\infty$, $G(\mu)=1$, and the model distribution (3) reduces to the sum of two isotropic Maxwellians with different temperatures and number densities. On the other hand, when $\Delta=0$, the model distribution (3) depicts a component with a complete one-sided loss cone, in the sense that the particle number density at $\mu=1$ is 0 . Thus it is expected that, in general, the excitation of unstable modes will tend to increase the value of $\Delta$, so as to fill up the loss cone and remove the source of instability. Indeed, quasilinear analysis that follows in the next section shows this behavior. The parameter $\delta$ controls the angle of the loss cone. One can easily compute the loss-cone angle by simply taking the second derivative of Eq. (3) with respect to $\mu$, and setting it equal to zero. This gives

$$
\cos \theta_{L C}=1+\delta \ln (2-\sqrt{3})
$$

Figure 1 shows the three-dimensional surface and contour plots of the energetic component of the particle distribution, $f_{e}(u, \mu)=\left(\pi^{3 / 2} \alpha^{3}\right)^{-1} \exp \left(-u^{2} / \alpha^{2}\right) G(\mu)(Z$ axis), of the model distribution function [see Eq. (3)] versus two normalized momentum components, $u_{\|}=u \mu$ (denoted in the figure by $X$ ) and $u_{\perp}=u\left(1-\mu^{2}\right)^{1 / 2}$ (denoted by $Y$ ), for energetic electron temperature $T=$ $10 \mathrm{keV}$ [corresponding to $\alpha=\left(2 T / m c^{2}\right)^{1 / 2}=0.2, m c^{2}$ being $500 \mathrm{keV}$ ]; loss-cone angle $\theta_{L C}=30^{\circ}$ [corresponding $\delta$ given by $\left.\delta=\left(\cos \theta_{L C}-1\right) / \ln (2-\sqrt{3})=0.1017\right]$; and $\Delta=0$ (complete loss cone). This choice of parameters is made so that direct comparison can be made with Ref. [24]. In Ref. [24], an additional choice of parameters, namely, that of the background electron temperature $T_{0}=100 \mathrm{eV}$ [corresponding to $\alpha_{0}=\left(2 T_{0} / m c^{2}\right)^{1 / 2}=$ 0.02 ], and the ratio of energetic to background electron

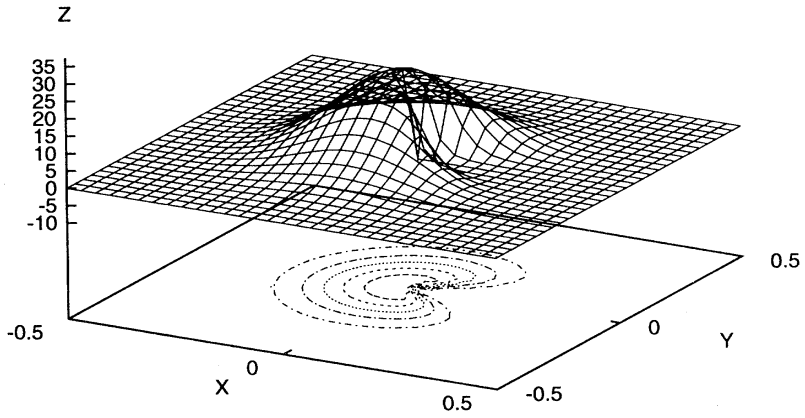

FIG. 1. Three-dimensional surface and two-dimensional contour plots of the model distribution function $f(u, \mu)$ (only the energetic component is shown - see the text). The $X$ axis stands for $u_{\perp}=u\left(1-\mu^{2}\right)^{1 / 2}, Y$ axis is $u_{\|}=u \mu$, and the $Z$ axis is the distribution function. The parameters used in the figure are $\alpha=0.2, \delta=0.1017$, and $\Delta=0$.

number density, $n_{e} / n_{0}=0.01$, is also made. Although we do not show the background component in Fig. 1, in the subsequent linear and quasilinear analysis we shall adopt such values for these additional parameters. Here, $\perp$ and $\|$ are defined with respect to the ambient magnetic field vector.

Using the model distribution function (3), one can show that the growth rate (2) reduces to

$$
\begin{aligned}
\Gamma(\omega, \theta)=\frac{\gamma_{\mathbf{k}}}{\Omega}= & -\frac{2 \sqrt{\pi}}{R} \frac{\omega_{p}^{2}}{\Omega^{2}} \sum_{n=1}^{\infty}\left(\Theta(n \Omega-\omega) \int_{-1}^{1} d \mu P_{n}^{+}(\mu)\right. \\
& +\Theta(\omega-n \Omega) \Theta\left(1-\mu_{n}^{2}\right) \\
& \left.\times \sum_{+,-} \int_{\mu_{n}}^{1} d \mu P_{n}^{ \pm}(\mu)\right)
\end{aligned}
$$

where $\Theta(x)$ is the step function; $\Theta(x)=1$ for $x>0$ and $\Theta(x)=0$ otherwise, and

$$
\begin{aligned}
P_{n}^{ \pm}(\mu)= & \frac{\left\{\left[\sin \theta+T\left(\cos \theta-N u_{ \pm} \mu\right)\right] J_{n}\left(b_{ \pm}\right)+(\Omega / \omega) b_{ \pm} J_{n}^{\prime}\left(b_{ \pm}\right)\right\}^{2}}{\left|u_{ \pm}-N \mu \cos \theta\right|} \\
& \times\left\{\frac{1}{\alpha_{0}^{3}} \frac{u_{ \pm}^{2}}{\alpha_{0}^{2}} \exp \left(-\frac{u_{ \pm}^{2}}{\alpha_{0}^{2}}\right)+\frac{n_{e}}{n_{0}} \frac{1}{\alpha^{3}(1+\Delta-\delta \tanh 1 / \delta)} \exp \left(-\frac{u_{ \pm}^{2}}{\alpha^{2}}\right)\right. \\
& \left.\times\left[\Delta \frac{u_{ \pm}^{2}}{\alpha^{2}}+\tanh \frac{1-\mu}{2 \delta}\left(\frac{N u_{ \pm} \cos \theta-\mu}{2 \delta} \operatorname{sech}^{2} \frac{1-\mu}{2 \delta}+\frac{u_{ \pm}^{2}}{\alpha^{2}} \tanh \frac{1-\mu}{2 \delta}\right)\right]\right\} \\
\mu_{n}= & \frac{\sqrt{2}}{N \cos \theta}\left(1-\frac{n \Omega}{\omega}\right)^{\frac{1}{2}}, \\
u_{ \pm}= & N \cos \theta\left[\mu \pm\left(\mu^{2}-\mu_{n}^{2}\right)^{\frac{1}{2}}\right] \\
b_{ \pm}= & \frac{\omega}{\Omega} N u_{ \pm}\left(1-\mu^{2}\right)^{\frac{1}{2}} \sin \theta .
\end{aligned}
$$

In Fig. 2, the growth rate (4) ( $Z$ axis) is plotted against the normalized real frequency $\omega / \Omega(X$ axis $)$ and propagation angle $\theta$ ( $Y$ axis) in three-dimensional surface and contour plot formats. The physical parameters are chosen exactly as in Fig. 1 (that is, $\alpha_{0}=0.02, \alpha$ $=0.2, \delta=0.1017, n_{e} / n_{0}=0.01$, and $\left.\Delta=0\right)$, with an additional parameter, $\omega_{p} / \Omega=0.1$. Again, the choice of $\omega_{p} / \Omega=0.1$ is made so that the present result can be directly compared with that of Ref. [24]. In the figure, the $Z$ axis denotes the normalized growth rate $\gamma_{\mathbf{k}} / \Omega$, the $X$ axis denotes the normalized frequency $\omega / \Omega$, and the $Y$ axis corresponds to the propagation angle $\theta$ (in degrees). In the calculation, we have kept up to $n=3$ terms in the Bessel function series. Specifically, plotted in the figure 
are the growth rates corresponding to the fundamental $\mathrm{X}$ mode [denoted by X1 - Fig. 2(a)], fundamental $\mathrm{O}$ mode [designated as $\mathrm{O} 1$ - Fig. 2(b)], and the second harmonic $\mathrm{X}$ mode [denoted by X2 - Fig. 2(c)]. The $\mathrm{Z}$ mode is stable in this case. Clearly, the most dominant mode is the X1 mode, in agreement with Ref. [24], in which it is assumed at the outset that the $\mathrm{X} 1$ mode dominates over other modes. The maximum growth rate for the X1 mode is an order of magnitude larger than that for the O1 mode, which in turn is larger than that of X2.
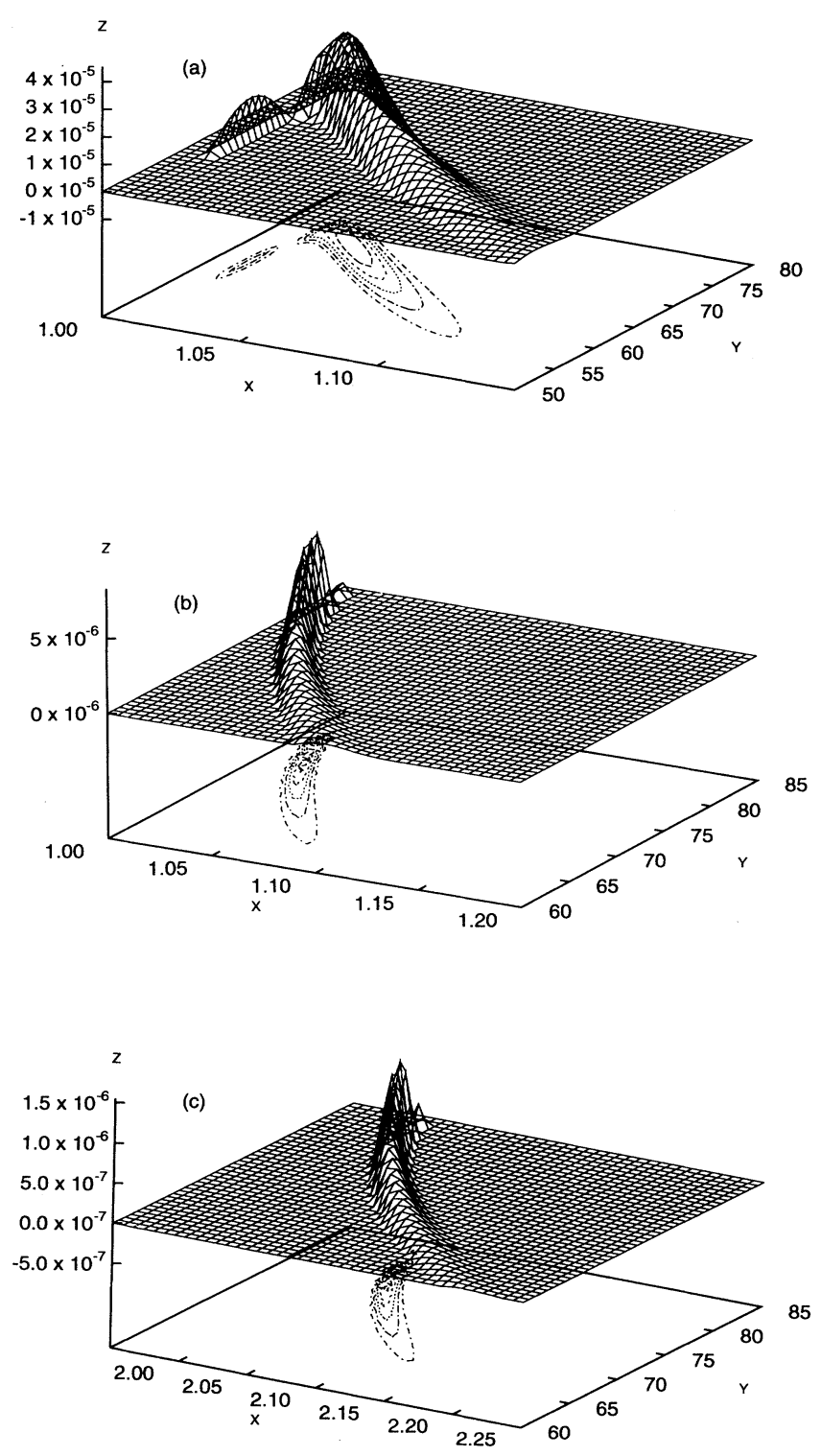

FIG. 2. Linear growth rates for (a) fundamental $\mathrm{X}$ mode (X1); (b) fundamental $\mathrm{O}$ mode (O1); and (c) second harmonic $\mathrm{X}$ mode (X2). The $X$ axis stands for the normalized frequency $\omega / \Omega, Y$ axis is for the angle $\theta$ (in degrees), and the $Z$ axis is the growth rate $\gamma_{\mathbf{k}} / \Omega$. The physical parameters are $n_{e} / n_{0}=$ $0.01, \omega_{p} / \Omega=0.1, \alpha_{0}=0.02, \alpha=0.2, \delta=0.1017$, and $\Delta=0$. Note that the $\mathrm{X} 1$ mode growth rate is an order of magnitude higher than the rest.
It is known that, among the various physical parameters, the most sensitive parameter that determines the relative importance of various unstable modes is the frequency ratio $\omega_{p} / \Omega$. Therefore we now consider the effect of variation on this parameter. Specifically, we hold other parameters fixed, but increase $\omega_{p} / \Omega$ from 0.1 to 0.2 . The results are shown in Fig. 3. Figure 3 is in the same format as Fig. 2 ( $Z$ axis denotes $\gamma_{\mathbf{k}} / \Omega, X$ axis denotes $\omega / \Omega$, and $Y$ axis corresponds to $\theta$ ). Note that Ref. [24] considers only the case of $\omega_{p} / \Omega=0.1$. It turns out that in the case of $\omega_{p} / \Omega=0.2$ the three most important unstable modes are the fundamental $\mathrm{X}$ and $\mathrm{O}$ modes (denoted by $\mathrm{X}$ and $\mathrm{O}$, respectively), and the $\mathrm{Z}$ mode. The growth
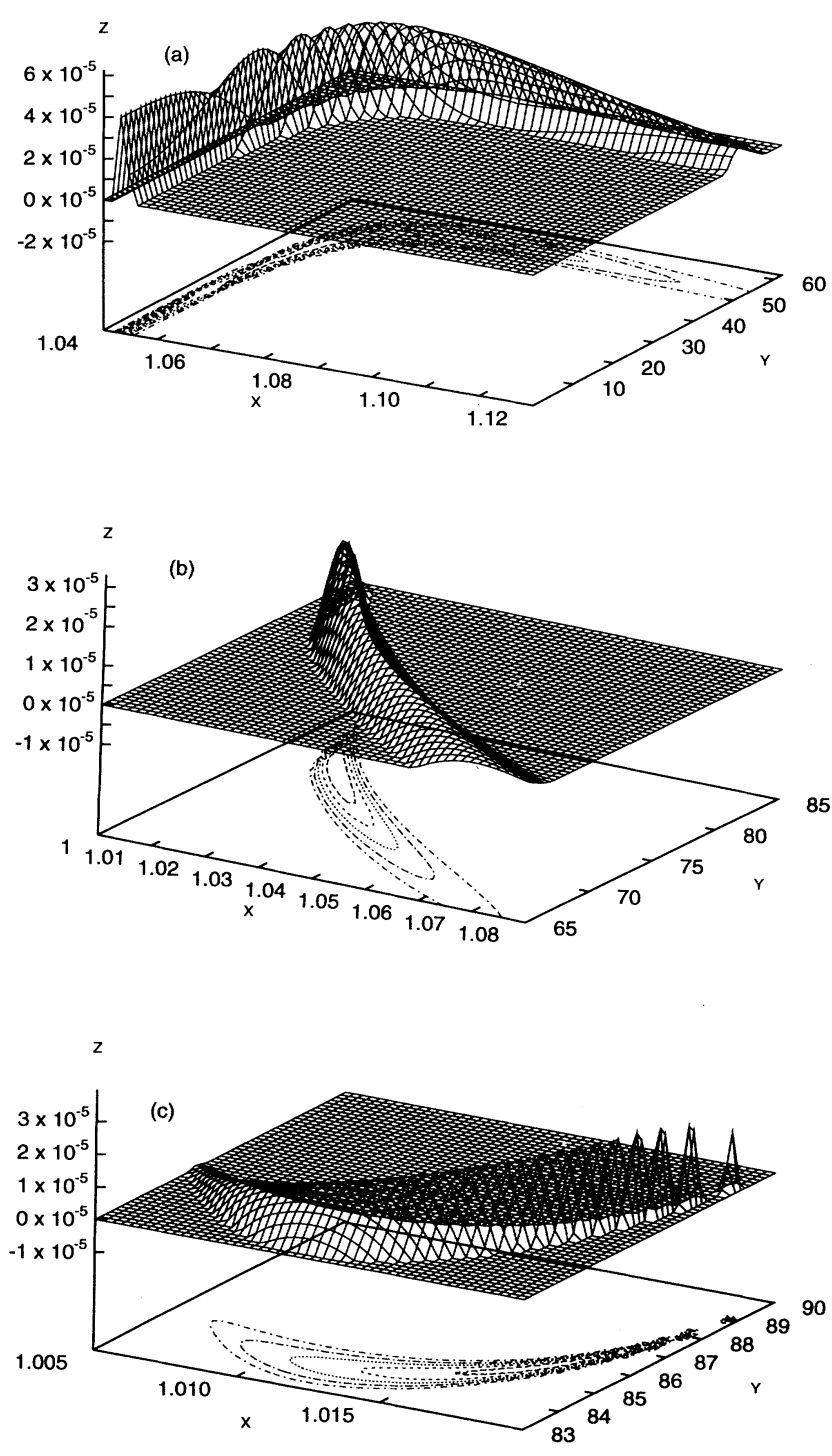

FIG. 3. Linear growth rates for (a) fundamental $X$ mode; (b) fundamental $\mathrm{O}$ mode; and (c) the $\mathrm{Z}$ mode. Axis labels are the same as in Fig. 2. The parameters used in the figure are the same as in Fig. 2, except $\omega_{p} / \Omega=0.2$. Note that in this case, all three modes have comparable growth rates, with the $\mathrm{X}$ mode growing at about twice the $\mathrm{Z}$ mode, which is in turn growing at a somewhat faster rate than the $\mathrm{O}$ mode. 
rate for the $\mathrm{X}$ mode is shown in Fig. 3(a), Fig. 3(b) corresponds to the $O$ mode growth rate, and finally, the $Z$ mode growth rate is shown in Fig. 3(c). The maximum growth rates for all three modes are comparable, with the $\mathrm{X}$ mode growth rate about twice that of the other two. Based upon this behavior, one might be tempted to conclude that the $\mathrm{X}$ mode is relatively the most important mode, and that in the nonlinear stage, the $\mathrm{X}$ mode will saturate to the highest wave intensity. However, such an intuitive conclusion proves to be premature. As we will show in the next section, among the three modes, the $\mathrm{X}$ mode does amplify at the fastest rate, but in the saturation stage it is the $\mathrm{Z}$ mode which reaches the highest level of intensity.

\section{QUASILINEAR STAGE OF MASER INSTABILITY}

The model distribution function (3) can be used in the study of nonlinear evolution of the maser instability in the following sense. Although Eq. (3) is not a solution to the particle kinetic equation in a rigorous sense, we nevertheless can approximate the nonlinear stage of the particle evolution by allowing the parameters $\alpha$ and $\Delta$ to evolve in time, while maintaining the essential functional form as described by Eq. (3). The primary motivation for such an approximate approach is the simplicity, which is based upon a reasonable physical ground. Putting it another way, we impose a self-similar form of the solution. The parameters $\alpha$ and $\Delta$ are related to appropriate moments of the particle distribution (3) itself. Thus, by taking appropriate moments of the kinetic equation for $f(u, \mu, t)$, one can construct the evolution equations for $\alpha(t)$ and $\Delta(t)$. Such a scheme is known as the moment method [33]. In what follows, we shall consider that the change in the background temperature during the quasilinear evolution process is minimal so that we can hold $\alpha_{0}$ constant in time. Moreover, the parameter $\delta$, which determines the loss-cone angle, is not expected to vary as a result of the wave process, since the loss-cone angle is determined by global configuration of the magnetic field inhomogeneity. Thus, within the context of uniform medium theory (which is employed in the present analysis), we may assume that $\delta$ is also a constant.

The quasilinear particle kinetic equation is well known, and it is given by

$$
\begin{aligned}
\frac{\partial f}{\partial t}= & \frac{1}{u^{2}}\left[u^{2}\left(D_{u u} \frac{\partial f}{\partial u}-\frac{D_{u \mu}}{u} \frac{\partial f}{\partial \mu}\right)\right] \\
& -\frac{1}{u} \frac{\partial}{\partial \mu}\left(D_{\mu u} \frac{\partial f}{\partial u}-\frac{D_{\mu \mu}}{u} \frac{\partial f}{\partial \mu}\right) \\
D_{a b}= & \frac{\pi e^{2}}{m^{2} c^{4} u^{2}} \sum_{n=1}^{\infty} \int d^{3} k\left|\mathbf{e}_{\mathbf{k}} \cdot \mathbf{V}_{n}^{*}\right|^{2} \frac{\delta E_{\mathbf{k}}^{2}}{\omega} \\
& \times \delta\left(1+\frac{u^{2}}{2}-\frac{n \Omega}{\omega}-N u \mu \cos \theta\right) \Delta_{a} \Delta_{b} \\
& \Delta_{u}=1, \quad \Delta_{\mu}=\mu-N u \cos \theta
\end{aligned}
$$

where

$$
\begin{aligned}
\left|\mathbf{e} \cdot \mathbf{V}_{n}^{*}\right|^{2}= & \frac{c^{2}}{\left(1+K^{2}+T^{2}\right) N^{2} \sin ^{2} \theta} \\
& \times\left([K \sin \theta+T(\cos \theta-N u \mu)] J_{n}(b)\right. \\
& \left.+\frac{\Omega}{\omega} b J_{n}^{\prime}(b)\right)^{2}
\end{aligned}
$$

In the above, $\mathbf{e}_{\mathbf{k}}=\delta \mathbf{E}_{\mathbf{k}} /\left|\delta E_{\mathbf{k}}\right|$ is the unit electric field vector for the spectral electric field component $\delta \mathbf{E}_{\mathbf{k}}$. Similarly, the wave kinetic equation is given by

$$
\frac{\partial}{\partial t} \delta E_{\mathbf{k}}^{2}=2 \gamma_{\mathbf{k}} \delta E_{\mathbf{k}}^{2}
$$

From Eq. (6), the following equations for appropriate moments can be readily derived:

$$
\begin{gathered}
\frac{d}{d t}\left\langle u^{2}\right\rangle=-2 \int d^{3} u u\left(D_{u u} \frac{\partial f}{\partial u}-\frac{D_{u \mu}}{u} \frac{\partial f}{\partial \mu}\right) \\
\frac{d}{d t}\langle\mu\rangle=\int d^{3} u \frac{1}{u}\left(D_{\mu u} \frac{\partial f}{\partial u}-\frac{D_{\mu \mu}}{u} \frac{\partial f}{\partial \mu}\right) .
\end{gathered}
$$

For the present purpose, a set of parameters $(\omega, \theta)$ is more natural than $\mathbf{k}$. Therefore the following relation is useful for our purpose:

$$
\begin{aligned}
\int d^{3} k \delta E_{\mathbf{k}}^{2}= & 2 \pi \int_{-1}^{1} d \cos \theta \int_{0}^{\infty} d \omega \frac{\omega^{2} \Omega}{c^{3}} \delta E^{2}(\omega, \theta) \\
& \times \frac{R}{\left(1+T^{2}\right) N \sin ^{2} \theta}
\end{aligned}
$$

Before we move on, let us introduce the following normalized time variable and normalized spectral wave energy density:

$$
\tau=\Omega t, \quad \mathcal{E}(\omega, \theta)=8 \pi \frac{\Omega^{3}}{c^{3}} \frac{\delta E^{2}(\omega, \theta)}{B_{0}^{2}}
$$

It is also interesting to note that the ratio of the wave energy density to particle energy density can be expressed as 


$$
\begin{aligned}
\frac{\mathcal{E}_{\text {wave }}}{\mathcal{E}_{\text {particle }}} & =\frac{\int d^{3} k\left(\delta E_{\mathbf{k}}^{2} / 8 \pi\right)\left[1+N^{2}\left(1-|\hat{\mathbf{k}} \cdot \hat{\mathbf{e}}|^{2}\right)\right]}{n_{0} m c^{2} \int d^{3} u\left(u^{2} / 2\right) f(u, \mu)} \\
& =\frac{8 \pi}{3} \frac{\Omega^{2}}{\omega_{p}^{2}} \frac{1}{\alpha_{0}^{2}+\left(n_{e} / n_{0}\right) \alpha^{2}} \int_{0}^{1} d \cos \theta \int_{0}^{\infty} d \omega \mathcal{E}(\omega, \theta) \frac{\omega R}{\left(1+T^{2}\right) N \sin ^{2} \theta}\left(1+N^{2} \frac{1+T^{2}}{1+K^{2}+T^{2}}\right) .
\end{aligned}
$$

In the above $\hat{\mathbf{k}}=\mathbf{k} /|\mathbf{k}|$.

Finally, we note that the following relations can be established from the model distribution function Eq. (3):

$$
\begin{array}{r}
\frac{d}{d \tau}\left\langle u^{2}\right\rangle=3 \frac{n_{e}}{n_{0}} \alpha \frac{d \alpha}{d \tau}, \\
\frac{d}{d \tau}\langle\mu\rangle \simeq \frac{n_{e}}{n_{0}} \frac{\delta}{(1+\Delta)^{2}} \frac{d \Delta}{d \tau} .
\end{array}
$$

From these, the moment kinetic equation (8) and the wave kinetic equation (7) can now be expressed in terms of various normalized quantities as follows:

$$
\begin{aligned}
\frac{d}{d \tau} \alpha(\tau) & =-\frac{2}{3 \alpha(\tau)} \frac{n_{0}}{n_{e}} \frac{\Omega^{2}}{\omega_{p}^{2}} \int_{0}^{1} d \cos \theta \int_{0}^{\infty} d \omega S(\omega, \theta) \Gamma[\omega, \theta \mid \alpha(\tau), \Delta(\tau)] \mathcal{E}(\omega, \theta, \tau), \\
\frac{d}{d \tau} \Delta(\tau) & =-\frac{[1+\Delta(\tau)]^{2}}{\delta} \frac{n_{0}}{n_{e}} \frac{\Omega^{2}}{\omega_{p}^{2}} \int_{0}^{1} d \cos \theta \int_{0}^{\infty} d \omega S(\omega, \theta) \eta[\omega, \theta \mid \alpha(\tau), \Delta(\tau)] \mathcal{E}(\omega, \theta, \tau), \\
\frac{\partial}{\partial \tau} \mathcal{E}(\omega, \theta, \tau) & =2 \Gamma[\omega, \theta \mid \alpha(\tau), \Delta(\tau)] \mathcal{E}(\omega, \theta, \tau),
\end{aligned}
$$

where $\Gamma$ is defined in Eq. (4). Moreover,

$$
S(\omega, \theta)=\frac{R(\omega, \theta)}{\left(1+K^{2}+T^{2}\right) N \sin ^{2} \theta}\left[\frac{\omega}{\Omega}+\frac{1}{2} \frac{T \omega_{p}^{2} \cos \theta}{(T \omega-\Omega \cos \theta)^{2}}\left(1+\frac{1-T^{2}}{1+T^{2}} \frac{\omega^{2}+\omega_{p}^{2}}{\omega^{2}-\omega_{p}^{2}}\right)\right],
$$

where $R(\omega, \theta)$ is defined in Eq. (3), and

$$
\begin{aligned}
\eta[\omega, \theta \mid \alpha(\tau), \Delta(\tau)] & =-\frac{2 \sqrt{\pi}}{R(\omega, \theta)} \frac{\omega_{p}^{2}}{\Omega^{2}} \sum_{n=1}^{\infty}\left(\Theta(n \Omega-\omega) \int_{-1}^{1} d \mu Q_{n}^{+}(\mu)+\Theta(\omega-n \Omega) \Theta\left(1-\mu_{n}^{2}\right) \int_{\mu_{n}}^{1} d \mu \sum_{+,-} Q_{n}^{ \pm}(\mu)\right) \\
Q_{n}^{ \pm}(\mu) & =\frac{N u_{ \pm} \cos \theta-\mu}{u_{ \pm}^{2}} P_{n}^{ \pm}(\mu)
\end{aligned}
$$

where $P_{n}^{ \pm}(\mu)$ is defined in Eq. (4).

In the numerical scheme employed in the present paper, we assume that the initial wave energy contained in each unstable mode is the same. We assume that the initial wave spectrum $\mathcal{E}(\omega, \theta, 0)$ is constant over an initially unstable domain. Then we assign the value for each wave mode in such a way that the total wave energy [integrated over $\omega$ and $\theta$ according to Eq. (9)] is the same for all the unstable modes. Specifically, we set $\mathcal{E}_{\text {wave }}(0) / \mathcal{E}_{\text {particle }}(0)$ $=1 \times 10^{-4}$ for all the modes. We then proceed to numerically solve Eq. (11). We consider two sets of parameters corresponding to Figs. 2 and 3. For the first case (corresponding to the initial set of parameters exactly the same as in Fig. 2), the results of the quasilinear moment calculation are displayed in Fig. 4. In the figure, the quantity $\alpha(\tau)$ is plotted against the normalized time $\tau$ in the top panel. The second panel shows the evolution of $\Delta(\tau)$ versus $\tau$. Finally, in the bottom panel, the total wave energy densities (integrated over $\omega$ and $\theta$ ) corresponding to $\mathrm{X} 1, \mathrm{O} 1$, and $\mathrm{X} 2$ modes are shown versus $\tau$ in loga- rithmic scale. The curve that saturates to the highest level is that of X1. The middle curve corresponds to O1, and the curve with the lowest saturation level is that of $\mathrm{X} 2$. In this case, the quasilinear saturation level generally follows the trend indicated by the initial growth rate for each mode. That is, the mode that has the highest level of initial growth rate saturates to the highest wave level. These results are again in good agreement with those obtained in Ref. [24].

Figure 5 shows the run with the second set of parameters (i.e., those of Fig. 3). The figure is in the same format as Fig. 4. The reader may recall that this run is for a higher value of the frequency ratio, $\omega_{p} / \Omega=0.2$ instead of 0.1 , while other parameters are held fixed as in Figs. 2 and 4. Recall also that initially the $\mathrm{X}, \mathrm{Z}$, and $\mathrm{O}$ modes all had comparable growth rates, but the $\mathrm{X}$ mode growth rate was about twice that of the rest. Consistent with this feature, it can be observed that the $\mathrm{X}$ mode grows at the fastest rate. However, a notable aspect of the present nonlinear calculation is that the $\mathrm{X}$ mode satu- 
rates at a somewhat lower intensity when compared with the $\mathrm{Z}$ mode. (Note that, unlike Fig. 4, the bottom panel is in linear scale.) The $\mathrm{O}$ mode is relatively unimportant when compared with the other two modes. Nevertheless, its saturation amplitude is non-negligible.

The reason the $\mathrm{Z}$ mode with lower growth rate nonlinearly dominates the $\mathrm{X}$ mode can be understood as follows. We consider, for instance, an $\mathrm{X}$ mode wave, with $\omega \simeq 1.05 \Omega$ and $\theta \simeq 30^{\circ}$ [see Fig. 3(a)]. The weakly relativistic resonance condition shows that the extremities of the resonance ellipse are $u_{+} \simeq 1.70$ and $u_{-} \simeq 0.028$. One of the extremities of the ellipse is well inside the loss-cone region, but most of the ellipse is located in velocity space where the density of particles is negligible and does not contribute to growth. When the loss cone is partially filled, the relevant region of amplification is affected and
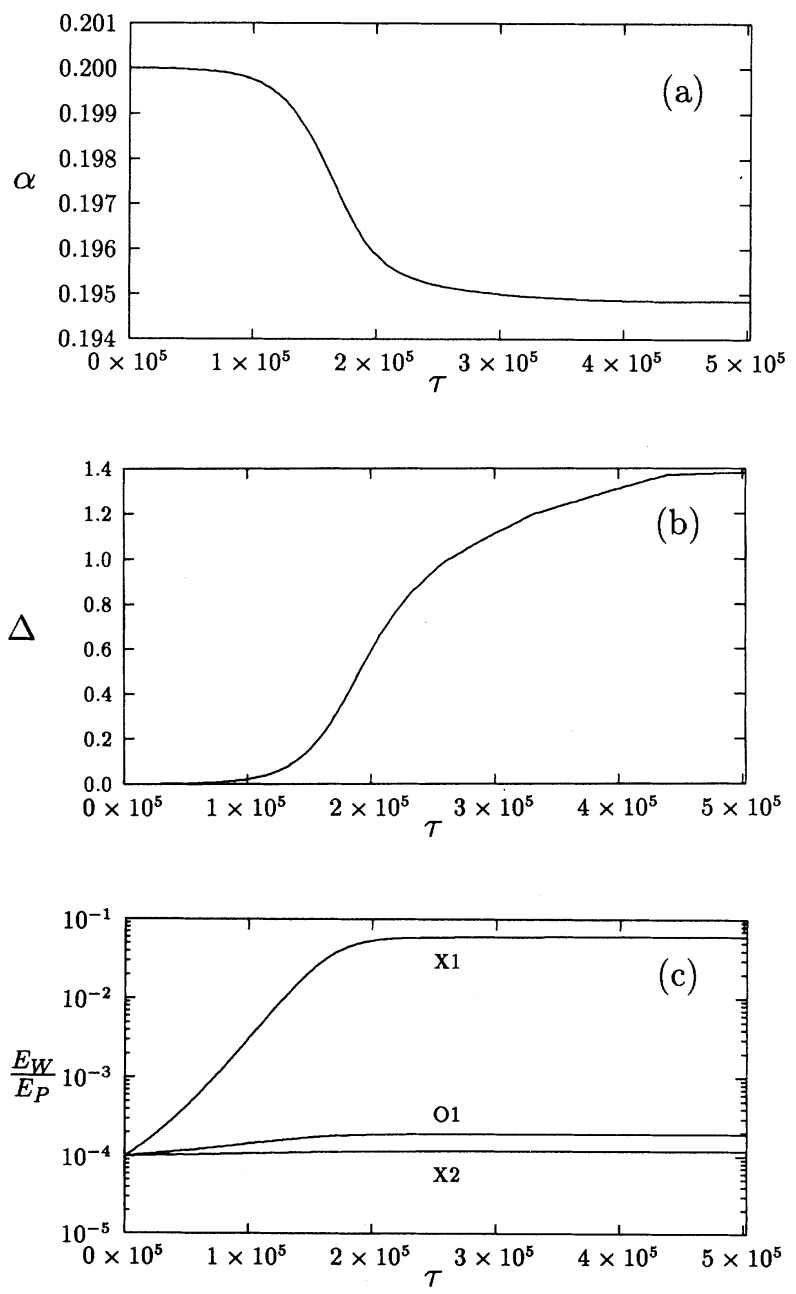

FIG. 4. Plot showing the evolution of (a) $\alpha(\tau)$, (b) $\Delta(\tau)$, and (c) the total (integrated over $\omega$ and $\theta$ ) normalized wave energy density for each mode (X1, O1, and X2). The initial parameters are exactly the same as used in Fig. 2. The normalization for the wave energy density is done over the particle energy density. Note that in (c) three curves are plotted in logarithmic scale. Consequently, the $\mathrm{O} 1$ and X2 modes have negligible wave energies compared to the $\mathrm{X} 1$ mode. the growth rate quickly disappears.

On the other hand, we consider a $\mathrm{Z}$ mode wave, with $\omega \simeq 1.015 \Omega$ and $\theta \simeq 80^{\circ}$ [see Fig. 3(c)]. The extremities of the resonant ellipse are now $u_{+} \simeq 0.29$ and $u_{-} \simeq 0.049$. The resonant ellipse is entirely in the loss cone region of velocity space. When the loss cone starts to be filled by quasilinear diffusion, the growth rate may decrease, but amplifying conditions remain longer than for the case of the $\mathrm{X}$ mode.

Finally, Fig. 6 shows the energetic component of the distribution function (see Fig. 1 for the description) at the end of the run shown in Fig. 4. For this case, at the time $\tau=5 \times 10^{5}, \alpha=0.1948$ and $\Delta=1.381$. The figure clearly shows the partially filled loss cones. For the run shown in Fig. 5, at $\tau=5 \times 10^{5}, \alpha=0.1949$ and $\Delta=$ 1.227. The final distribution is not shown because it is
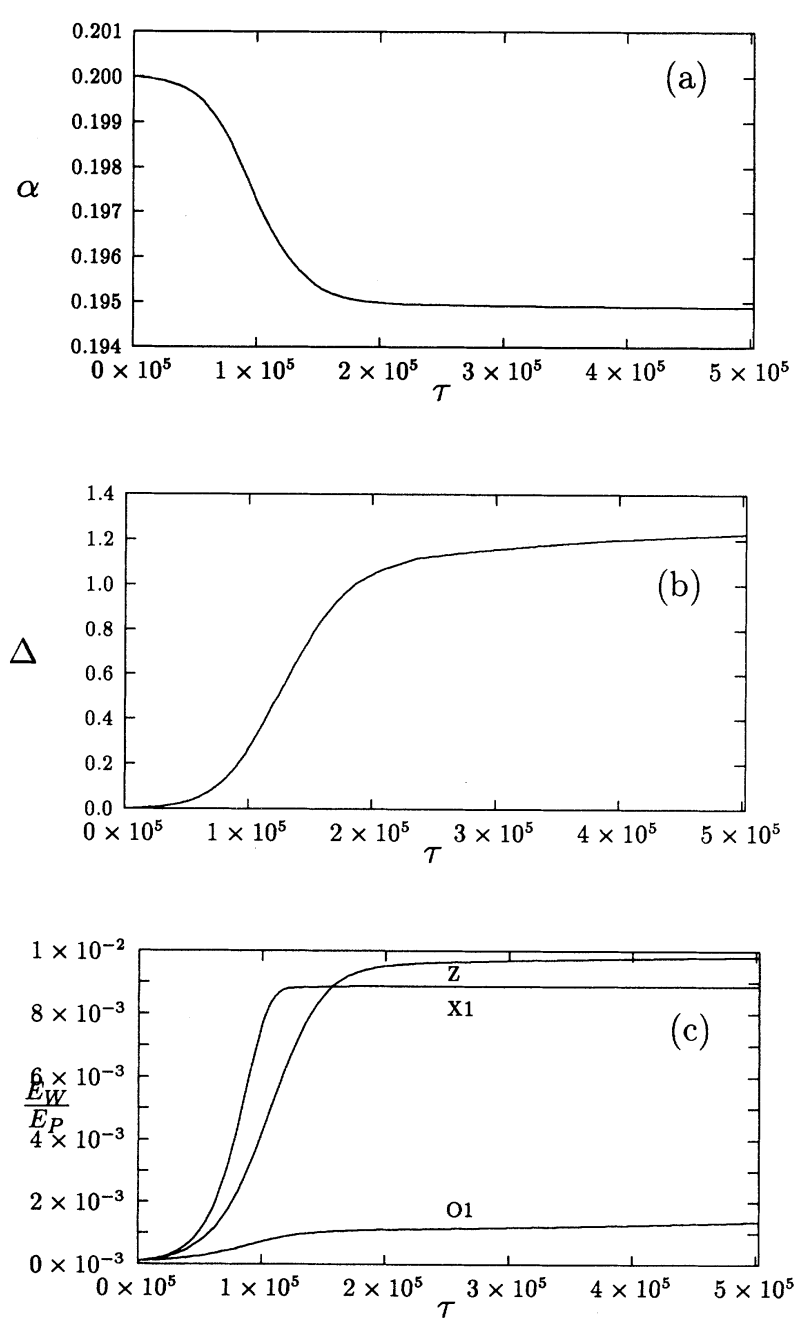

FIG. 5. The same as Fig. 4, except that this case corresponds to $\omega_{p} / \Omega=0.2$. [(a) is for $\alpha(\tau),(\mathrm{b})$ is for $\Delta(\tau)$, and (c) is for normalized wave energy densities for $\mathrm{X}, \mathrm{Z}$, and $\mathrm{O}$ modes.] Note that despite the fact that the $X$ mode initially grows the fastest, the $\mathrm{Z}$ mode has a higher saturation amplitude. Note also that, unlike Fig. 4, the vertical axis in panel (c) is in linear scale. 


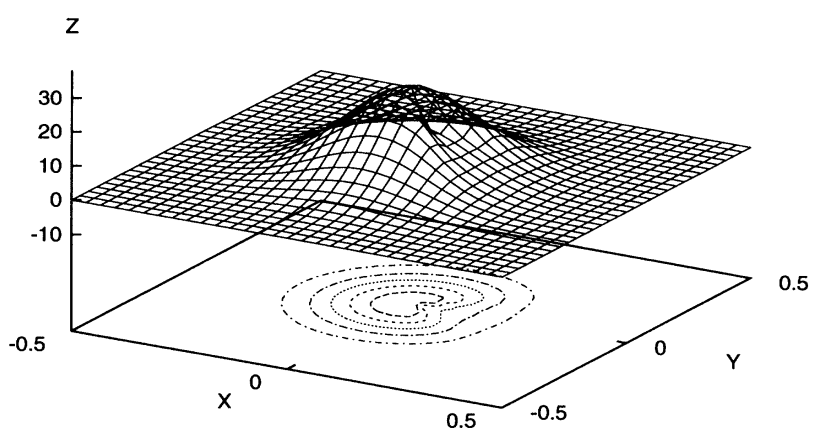

FIG. 6. The asymptotic energetic electron distribution function corresponding to Fig. 4.

very similar to that of Fig. 6 .

Notice that the asymptotic distributions still show some remaining loss-cone feature for the low-energy portion of the distribution, but such a feature is not sufficient to contribute to the wave growth. Note also that the final form of the distribution function compares very well with that obtained by full numerical solution in Ref. [24].

\section{SUMMARY AND CONCLUSIONS}

In the present paper, we have carried out a quasilinear analysis of the loss-cone driven electron cyclotron maser instability. The method is based upon an efficient moment calculation of the quasilinear kinetic equation. The difference of the present approach is that, instead of solving the quasilinear equation directly, we make a reasonable conjecture on the time dependency of the particle distribution function. Since the results of previous simulation studies confirm that the dominant nonlinear mechanism is the filling up of the loss cone, we model a self-similar form of the loss-cone distribution accordingly. This allows us to evaluate the evolution of the var- ious wave modes, as well as the particle moments, very efficiently. The result is then tested against the formal result obtained in Ref. [24] with the same set of parameters used in that paper. We then proceed to the case when the frequency ratio $\omega_{p} / \Omega$ is slightly larger. In this instance, unlike Ref. [24], the $X, O$, and $Z$ modes all have comparable growth rates. Our analysis shows that the competition among various unstable modes can be very important for this parameter regime. In particular, although the initial growth suggests that the $\mathrm{X}$ mode has the highest growth rate, in the nonlinear stage, the mode saturates to a lower wave level, when compared to the $\mathrm{Z}$ mode. Thus we conclude that the study of linear instability alone is not sufficient, and that nonlinear (or quasilinear) analysis is called for. However, a direct numerical solution of the fully self-consistent quasilinear equation including multiple unstable modes is very time consuming and costly in terms of computation time. Therefore it is our belief that the moment method introduced in this paper can serve the purpose of providing efficient and reasonable results.

\section{ACKNOWLEDGMENTS}

This work was supported in part by the Brazilian agencies Conselho Nacional de Desenvolvimento Científico e Tecnológico (CNPq) and Financiadora de Estudos e Projetos (FINEP). Numerical computations were performed in part at the Supercomputer Center located at the Universidade Federal do Rio Grande do Sul (CESUPUFRGS), and in part at the National Science Foundation Supercomputer Center located at the University of California, San Diego, through a resource grant made available to the University of Maryland. One of the authors (P.H.Y.) acknowledges the hospitality of the Institute of Physics at UFRGS and CESUP-UFRGS during his visit to Porto Alegre, Brazil.
[1] C. S. Wu and L. C. Lee, Astrophys. J. 230, 621 (1979).

[2] R. G. Hewitt, D. B. Melrose, and K. G. Rönmark, Proc. Astron. Soc. Aust. 4, 226 (1981).

[3] C. S. Wu, S. T. Tsai, M. J. Xu, and J. W. Shen, Astrophys. J. 248, 384 (1981).

[4] C. S. Wu, H. K. Wong, D. J. Gorney, and L. C. Lee, J. Geophys. Res. 87, 4476 (1982).

[5] H. K. Wong, C. S. Wu, F. J. Ke, R. S. Schneider, and L. F. Ziebell, J. Plasma Phys. 28, 503 (1982).

[6] D. B. Melrose and G. Dulk, Astrophys. J. 259, 844 (1982).

[7] N. Omidi and D. A. Gurnett, J. Geophys. Res. 87, 2377 (1982).

[8] J. S. Wagner, L. C. Lee, C. S. Wu, and T. Tajima, Geophys. Res. Lett. 10, 483 (1983).

[9] J. S. Wagner, L. C. Lee, C. S. Wu, and T. Tajima, Radio Sci. 19, 509 (1984).

[10] N. Omidi and D. A. Gurnett, J. Geophys. Res. 89, 10801 (1984).
[11] C. S. Wu and X. M. Qiu, J. Geophys. Res. 89, 883 (1984).

[12] D. Le Quéau, R. Pellat, and A. Roux, J. Geophys. Res. 89, 897 (1984).

[13] D. Le Quéau, R. Pellat, and A. Roux, Phys. Fluids 27, 247 (1984).

[14] D. B. Melrose, R. G. Hewitt, and G. A. Dulk, J. Geophys. Res. 89, 2831 (1984).

[15] P. L. Pritchett and R. J. Strangeway, J. Geophys. Res. 90, 9650 (1985).

[16] C. S. Wu, Space Sci. Rev. 41, 215 (1985).

[17] P. L. Pritchett, Phys. Fluids 29, 2919 (1986).

[18] L. F. Ziebell and G. Granata, Phys. Fluids 29, 3730 (1986).

[19] M. J. Aschwanden and A. O. Benz, Astrophys. J. 332, 447 (1988).

[20] L. F. Ziebell, J. Plasma Phys. 39, 431 (1988).

[21] P. Galopeau, P. Zarka, and D. Le Quéau, J. Geophys. Res. 94, 8739 (1989).

[22] P. L. Pritchett and R. M. Winglee, J. Geophys. Res. 94, 
129 (1989).

[23] P. H. Yoon and T. Chang, Astrophys. J. 343, 31 (1989).

[24] M. J. Aschwanden, Astron. Astrophys. 237, 512 (1990).

[25] M. Güdel, Astron. Astrophys. L1, 239 (1990).

[26] P. H. Yoon and C. S. Wu, Phys. Rev. A 44, 6819 (1991).

[27] R. Gaelzer, L. F. Ziebell, and R. S. Schneider, J. Geophys. Res. 97, 19299 (1992).

[28] R. Gaelzer, L. F. Ziebell, and R. S. Schneider, J. Geophys. Res. 99, 8905 (1994).

[29] N. A. Krall and A. W. Trivelpiece, Principles of Plasma
Physics (McGraw-Hill, New York, 1973).

[30] T. H. Stix, The Theory of Plasma Waves (McGraw-Hill, New York, 1962).

[31] D. B. Melrose, Plasma Astrophysics, Natural Processes in Diffuse Magnetized Plasmas (Gordon and Breach, New York, 1980), Vols. 1 and 2.

[32] L. F. Ziebell and P. H. Yoon, Phys. Plasmas (to be published).

[33] R. C. Davidson and J. M. Ogden, Phys. Fluids 18, 1045 (1975). 